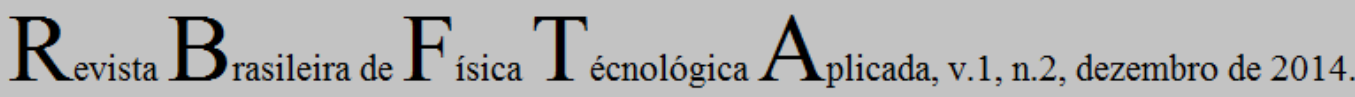

\section{Investigação sobre o funcionamento de um termômetro digital de baixo custo ${ }^{1}$}

\author{
La investigación sobre el funcionamiento de un termómetro digital de bajo coste
}

\author{
Research on the Operation of a Low-cost Digital Thermometer
}

Luciano Soares Pedroso ${ }^{2}$, Fabrício Pimenta Neto, Mauro Sérgio Teixeira de Araújo

Resumo: Apresenta-se nesse artigo o resultado de um ensaio investigativo em laboratório didático sobre o funcionamento de um Termômetro Digital construído com material de baixo custo e de fácil aquisição. Para a montagem do experimento utilizou-se um multímetro digital, bateria e um sensor de temperatura. O Termômetro digital apresentou algumas características adicionais, como robustez, facilidade de construção, baixo custo, e resposta rápida a variações de temperatura. Os resultados dos experimentos desenvolvidos comprovam a dependência linear da tensão medida no material em função da temperatura utilizado na construção do sensor de temperatura bem como precisão e rapidez na medida da temperatura, o que caracteriza um desempenho satisfatório.

Palavras-chave: termômetro digital ; temperatura; circuito integrado LM 35 DZ; termodinâmica.

Resumen: Se presenta en este artículo el resultado de un ensayo de investigación en un laboratorio didáctico sobre el funcionamiento de un Termómetro Digital construído con bajo coste y fácil adquisición. Para el montaje del experimento, se utilizó un multímetro digital, una batería y un sensor de temperatura. El Termómetro Digital presentó algunas características adicionales, tales como la robustez, facilidad de construcción, bajo coste, y respuesta rápida a las variaciones de temperatura. Los resultados de los experimentos desarrollados confirman una dependencia lineal de la tensión medida en el material resultante de la temperatura utilizada en la construcción del sensor de temperatura, así como precisión y medición rápida de la temperatura, que cuenta como un rendimiento satisfactorio.

Palabras clave: termómetro digital; temperatura; circuito integrado LM 35 DZ.; termodinámica.

Abstract: This article presents the results of an investigative essay, conducted in a didactic laboratory, which researches the performance of a Digital Thermometer built with a low-cost and easy to purchase material. For the formulation of the experiment, it is employed a digital multimeter, a battery, and a temperature sensor. The Digital Thermometer presents some additional features, such as hardiness, easiness of assembling, low-cost, and fast response to temperature variations. The results of the developed experiments substantiate the linear dependence of the measured voltage of the material employed, in relation to the temperature, in the assembling of the temperature sensor as well as the accurateness and speed of the temperature measurement, which embodies a satisfactory performance.

Keywords: digital thermometer; temperature; LM 35 DZ chip; thermodynamics.

\footnotetext{
${ }^{1}$ Trabalho apresentado ao XX Simpósio Nacional de Ensino de Física sob o título "Construção e Validação de um Termômetro Digital de Baixo Custo" - USP - São Paulo, 2013.

${ }^{2}$ Graduado com licenciatura em Física e Matemática, mestre em Ensino de Física e Doutor em Ensino de Ciências e Matemática. Atualmente é professor da FESP/UEMG, e-mail: luciano.pedroso@fespmg.edu.br
} 


\section{Introdução}

Um desafio dos professores que ministram as disciplinas de Ciências, Química, Física e Biologia é o de por em prática estudos teóricos relacionados à termodinâmica. Essa dificuldade, de forma especial, observa-se quando os experimentos necessitam de precisão e rapidez na leitura da temperatura. Medir a temperatura corretamente é muito importante em todos os ramos da ciência, pois muitas propriedades físicas dos materiais dependem dessa grandeza. Por exemplo, a fase do material, se ele é sólido, líquido ou gasoso, tem relação com sua temperatura. Também a densidade, a solubilidade, a pressão de vapor, a condutividade elétrica, entre outros, dependem da temperatura. Em outra circunstância, a temperatura do corpo humano, mantida em algo em torno de $37^{\circ} \mathrm{C}$, regula inúmeros processos biológicos e químicos.

Percebe-se que a medida e o controle da temperatura desempenham um papel muito importante, seja nas indústrias, nos laboratórios didáticos e científicos, na medicina e até mesmo em residências. Constantemente realizam-se medidas e controle da temperatura de grandes variedades de objetos e nas mais diversas circunstâncias. Para medir a temperatura de um corpo é preciso encontrar alguma propriedade que se relacione com a energia de agitação de seus átomos e moléculas. Partindo dessa propriedade, entende-se ser possível construir instrumentos (termômetros) que possibilitem medir a temperatura.

Nesse contexto, o que se propõe nesse trabalho é apresentar um termômetro digital desenvolvido com material de baixo custo e fácil aquisição e apresentar resultados de uma pesquisa sobre o seu funcionamento.

\subsection{Termômetros}

É interessante observar que a temperatura foi uma das primeiras grandezas da termodinâmica que pôde ser medida por Galileu em 1592 (HAYNIE, 2001) utilizando um termoscópio de ar. A partir do termoscópio de Galileu, diversos termômetros foram construídos. Em 1640, segundo o mesmo autor, o grande Duque Ferdinando II (1610-1670) da Toscana, um dos fundadores da Academia Florentina do Experimento, construiu o primeiro termômetro de álcool, cuja aplicação deu-se nas áreas de medicina, agricultura e meteorologia. E, em 1713, o alemão Daniel Gabriel Fahrenheit (1686-1736), operário de uma fábrica de vidro, também construiu um termômetro a álcool que logo depois foi substituído por um de mercúrio. O termômetro de Fahrenheit passou a trabalhar com pontos fixos de 
temperatura: a temperatura de ebulição da água (que ele definiu como sendo $212^{\circ} \mathrm{F}$ ) e o ponto de fusão do gelo $\left(32^{\circ} \mathrm{F}\right)$. Escala conhecida e utilizada até hoje. Numa tentativa de aperfeiçoamento e na obtenção de medidas mais precisas, o zoólogo francês René A. F. de Réaumur (1683-1757) e o astrônomo sueco Anders Celsius (1701-1744) estabeleceram novas escalas (HAYNIE, 2001). Em 1817, o relojoeiro francês Abraham Louis Breguet (1747-1823) (HAYNIE, 2001) construiu termômetros metálicos que deram origem aos termógrafos: termômetros registradores.

A construção de todos os termômetros acima citados baseou-se em dois princípios:

$\checkmark \quad \mathrm{O}$ aparelho entra em equilíbrio térmico com o sistema cuja temperatura se busca medir (lei zero da termodinâmica);

$\checkmark \quad$ Alguma grandeza física do elemento sensor é afetada pela temperatura (o volume do líquido, no caso dos termômetros por princípio de dilatação; a resistência elétrica; a diferença de potencial, dentre outros).

Há, todavia, termômetros construídos a partir de princípios diferentes. Um dos princípios que pode ser utilizado está em medir a energia irradiada pelo objeto que está aquecido.

\subsection{Medida de temperatura}

De acordo com Halliday, Resnick e Walker (2003 e 2012), existem diversas grandezas físicas que se modificam quando é variada a percepção fisiológica de temperatura. Entre essas grandezas estão a expansão volumétrica de líquidos e gases, a dilatação linear de lâminas bimetálicas, a resistividade elétrica de materiais condutores, a diferença de potencial elétrico entre as junções de um termopar, a intensidade e comprimento de onda da radiação emitida por um corpo (principalmente radiação infravermelha) e até mesmo a cor do filamento de uma lâmpada. Qualquer uma destas grandezas pode ser usada para a construção de um termômetro e a indicação de uma escala termométrica.

A definição de uma escala termométrica é feita admitindo-se uma relação monotônica contínua entre a propriedade termométrica da substância escolhida e a temperatura medida em uma escala conhecida. A escolha de uma substância e de sua propriedade termométrica, juntamente com a relação admitida entre a propriedade e a temperatura, conduz a uma escala termométrica específica. 


\subsection{Componentes do termômetro digital de baixo custo}

Propõe-se uma maneira de medir a temperatura com precisão e rapidez utilizando material de baixo custo e fácil aquisição. Para tanto, utilizou-se um multímetro digital, um circuito integrado LM 35 DZ e uma bateria de 9,0 volts. O sensor de temperatura LM35 possui um comportamento linear da tensão em $\mathrm{mV}$ em função da temperatura em ${ }^{\circ} \mathrm{C}(+10$ $\left.\mathrm{mV} /{ }^{\circ} \mathrm{C}\right)$, não necessita de calibração e possui uma baixa corrente de drenagem, da ordem de $60 \mu \mathrm{A}$, o que evita o rápido descarregamento da fonte (bateria) e seu auto-aquecimento (CIPELLI e SANDRINI, 2001).

O circuito integrado LM35 trabalha como um transistor, modulando a corrente de saída ao variar e tensão aplicada (BOYLESTAD e NASHELSKY, 1998) funcionando como um sensor de temperatura preciso, sensível, de baixo custo e fácil de encontrar. Esse circuito fornece a temperatura diretamente em graus Celsius. Cada $10 \mathrm{mV}$ na saída corresponde a um grau Celsius (BOYLESTAD e NASHELSKY, 1998). Ou seja, $471 \mathrm{mV}=47,10^{\circ} \mathrm{C}$, onde um multímetro barato serve como display. A saída do $\mathrm{LM} 35$ varia de $-55,00^{\circ} \mathrm{C}$ a $150,00^{\circ} \mathrm{C}$ com um erro de $\pm 0,75^{\circ} \mathrm{C}$. A Figura 1 mostra o esquema de ligação entre os componentes a fim de construir o termômentro desejado.

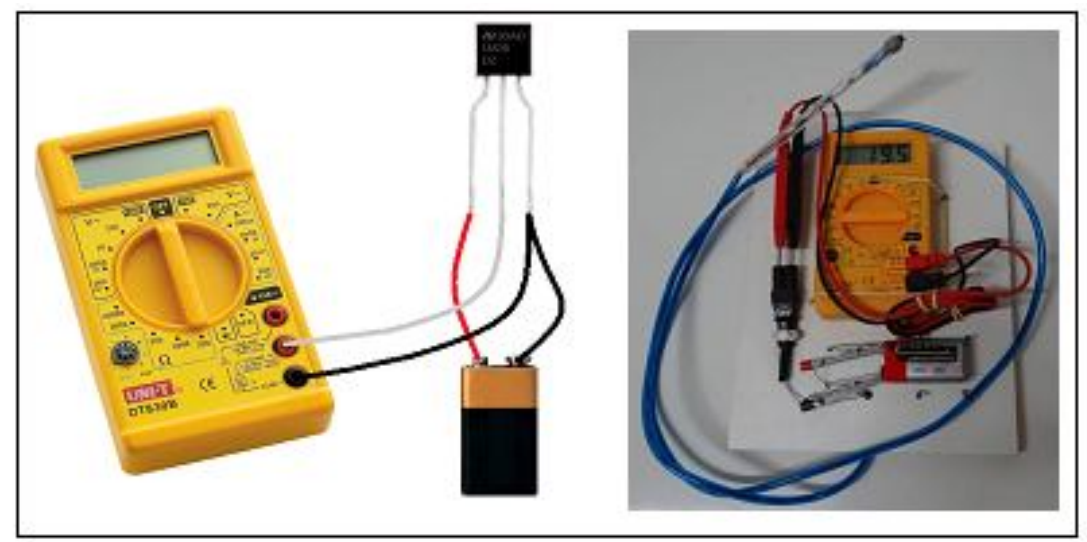

Figura 1: Termômetro digital de baixo custo, esquema da ligação e seus componentes.

Como se observa na Figura 1, para a construção do termômetro digital foram necessários os seguintes materiais: um transistor LM35DZ - Termômetro na forma de circuito integrado, uma bateria de 9 volts, duas garras jacaré para conectar a bateria de 9 volts (opcional), fio de cobre, multímetro ajustado para milivolts.

Para a construção, deve-se seguir os seguintes passos: soldar os fios no LM35 como mostrado na Figura 01, em seguida soldar ou conectar a ligação da pilha, prendendo os 
terminais do multímetro aos fios do circuito usando um conector. Após as conexões feitas, deve-se ajustar o multímetro para milivolts, na faixa de $2000 \mathrm{mV}$.

\section{Procedimentos para a calibragem do Termômetro Digital}

Inicialmente, coloca-se $2000 \mathrm{~g}$ de água a uma temperatura de 18 graus Celsius (medida no termômetro de Mercúrio) em contato com um ebulidor elétrico de 1000 watts de potência durante um tempo de 240 segundos.

A calibragem do termômetro digital foi realizada colocando-o próximo a um termômetro de Mercúrio, submersos à mesma altura em relação ao nível da água. Foram comparadas as leituras indicadas no termômetro digital e no termômetro de mercúrio a cada intervalo de 30 segundos.

Tabela 1: Resultados comparativos entre as leituras realizadas nos dois termômetros.

\begin{tabular}{|c|c|c|}
\hline Tempo (s) & Termômetro Digital $\left({ }^{\circ} \mathbf{C}\right)$ & Termômetro de Mercúrio $\left({ }^{\circ} \mathbf{C}\right)$ \\
\hline 0 & 18,2 & 18,0 \\
\hline 30 & 21,6 & 22,0 \\
\hline 60 & 25,2 & 26,0 \\
\hline 90 & 28,8 & 29,0 \\
\hline 120 & 32,5 & 34,0 \\
\hline 150 & 36,3 & 36,0 \\
\hline 180 & 39,6 & 40,0 \\
\hline 210 & 43,0 & 43,0 \\
\hline 240 & 46,8 & 47,0 \\
\hline
\end{tabular}

Constatou-se, com boa precisão, que a leitura indicada no termômetro digital está de acordo com a leitura realizada no termômetro de Mercúrio, conforme mostra a Tabela 01. 


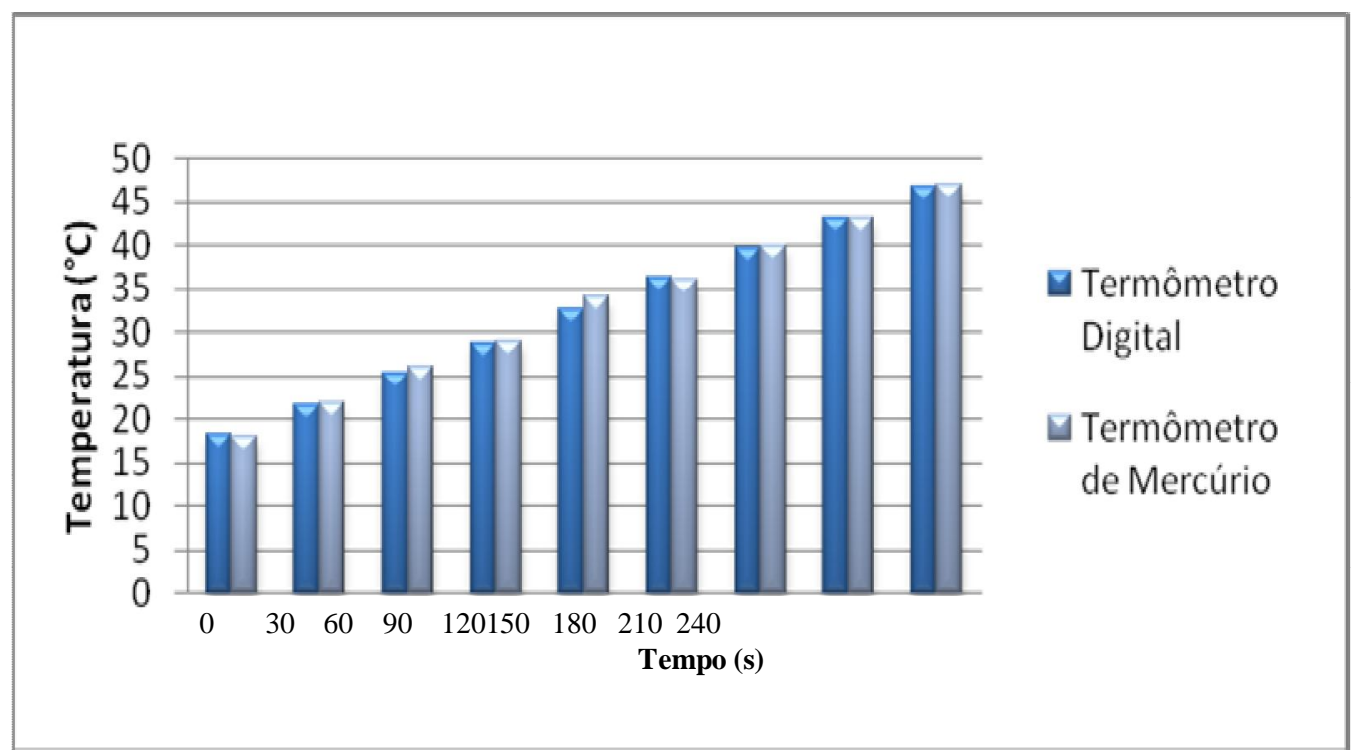

Figura 2: Gráfico da comparação dos resultados entre os dois termômetros utilizados, termômetro de mercúrio e termômetro digital.

Pelos gráficos da Figura 2 e Figura 3, certificou-se a linearidade do LM 35 DZ.

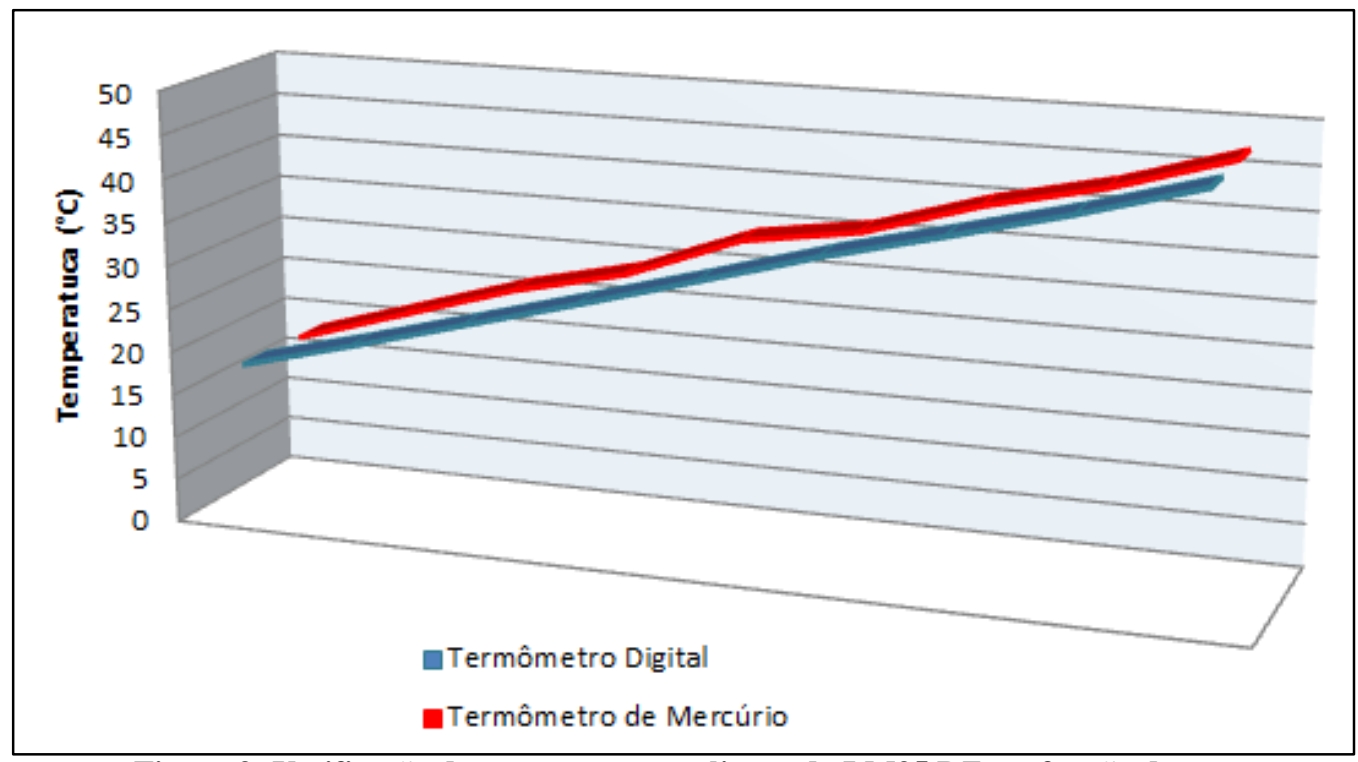

Figura 3: Verificação do comportamento linear do LM35 DZ em função da temperatura.

Observa-se na Figura 4 como se caracteriza a qualidade de resposta dada pelo termômetro digital em comparação ao termômetro de mercúrio em um tempo de 210 segundos. 


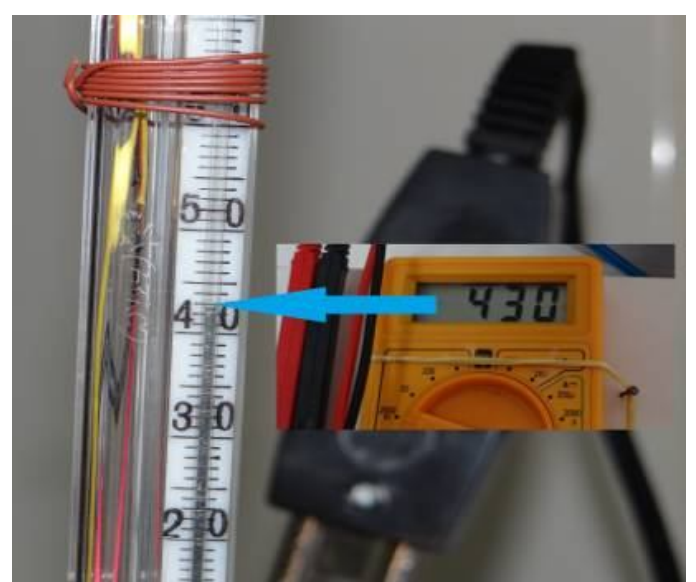

Figura 4: Indicação da leitura realizada no termômetro digital e no termômetro de Mercúrio no tempo de 210 segundos.

Constatou-se que o termômetro digital atingiu o equilíbrio térmico bem mais rápido que o termômetro de mercúrio, evidenciando sua utilização em experimentos em que a dissipação calórica é muito rápida.

\section{Procedimentos para validação da calibragem}

Utilizou-se um ebulidor elétrico de potência $1000 \mathrm{~W}$ imerso em um recipiente com 2,0 $\mathrm{kg}$ de água à temperatura inicial de $45^{\circ} \mathrm{C}$, conforme a Figura 05 . Ao final de 60 segundos constatou-se que a temperatura indicada pelo termômetro digital era de $52,4^{\circ} \mathrm{C}$. Como a troca de calor entre o ebulidor e a água foi rápida, foram consideradas desprezíveis as perdas de calor para o ambiente.

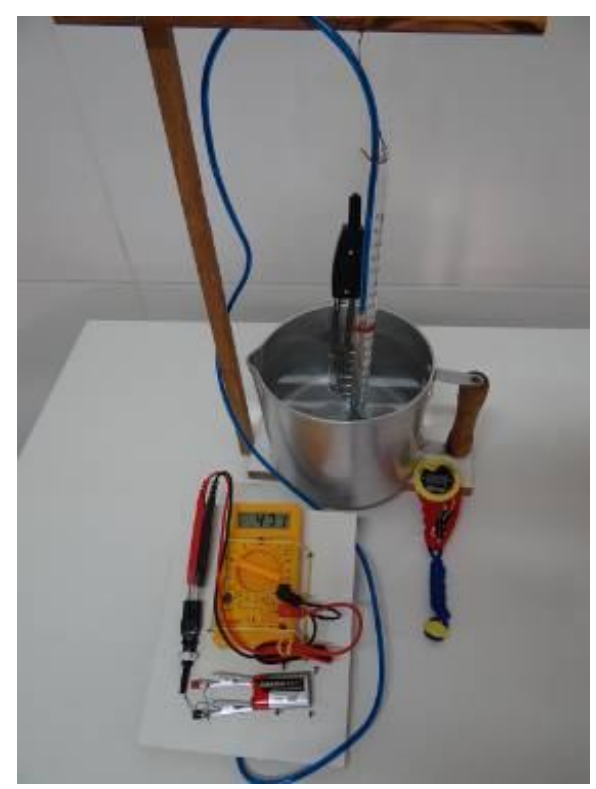

Figura 5: Aparato experimental. 
Partindo do princípio da conservação de energia, procedeu-se da seguinte forma: mergulhou-se o ebulidor de potência $1000 \mathrm{~W}$ em um recipiente contendo $2000 \mathrm{~g}$ de água à temperatura inicial de $45^{\circ} \mathrm{C}$ durante 60 segundos e após esse tempo registrou-se o valor obtido pelo termômetro digital e de mercúrio. Com o auxilio da equação da calorimetria (1) e dos valores anotados no inicio do experimento calculou-se a temperatura final obtida após o tempo de 60 segundos. Usou-se o valor tabelado para o calor específico da água $4190 \mathrm{~J} / \mathrm{kg} . \mathrm{K}$.

$$
\mathrm{Q}=\mathrm{m} \cdot \mathrm{c} \cdot \Delta \mathrm{T}
$$

O ebulidor forneceu ao sistema (água, termômetros e recipiente) $60 \mathrm{KJ}$ de energia durante os 60 segundos e fez com que a massa de água atingisse a $\mathrm{T}_{\mathrm{f}}=52,2^{\circ} \mathrm{C}$, temperatura determinada a partir do seguinte cálculo:

$$
(60 \mathrm{KJ})=(2,0 \mathrm{~kg}) \cdot(4190 \mathrm{~J} / \mathrm{kg} \cdot \mathrm{K}) \cdot\left(\mathrm{T}_{\mathrm{f}}-45\right)
$$

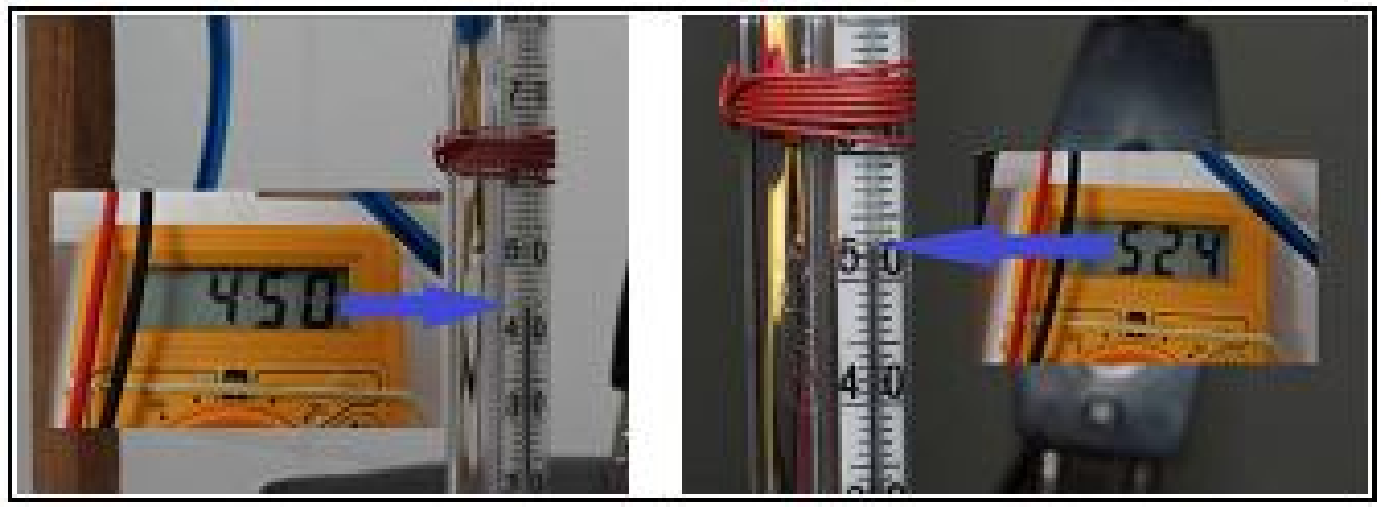

Figura 6: Temperatura inicial e final medida no experimento.

A Figura 6 (temperatura final) evidencia a qualidade de resposta do termômetro digital, no momento da medida da temperatura final de nosso experimento, indo de encontro ao resultado obtido nos cálculos acima.

\section{Considerações Finais}

A utilização do sistema proposto em atividades de ensino do termômetro digital de leitura direta apresenta vantagens significativas do ponto de vista prático em relação aos termômetros de Mercúrio. Inicialmente, deve-se considerar que o termômetro proposto apresenta uma resposta rápida, cerca de 2,0 segundos, em comparação ao termômetro convencional de Mercúrio que estabiliza a leitura em torno de 15,0 segundos, o qual utiliza o 
volume do líquido como propriedade termométrica. Outra vantagem no uso do termômetro proposto está no fato de permitir efetuar leituras diretamente em recipientes fechados ou em locais de difícil visualização. Para realizar esse tipo de leitura com um termômetro de Mercúrio faz-se necessário, muitas vezes, retirar parcialmente o termômetro da posição, o que pode resultar em dificuldades de leitura e/ou em erros nas medidas. Deve-se considerar também, a fragilidade dos termômetros de Mercúrio que pode resultar em quebras durante as aulas. A utilização do sistema proposto minimiza esse problema devido à robustez de sua estrutura.

\section{Referências Bibliográficas}

BOYLESTAD, R. L.; NASHELSKY, L. Dispositivos eletrônicos e teoria de circuitos. Rio de Janeiro: Livros Técnicos e Científicos, 1998.

CIPELLI, A. M. V.; SANDRINI, W. J. Teoria e desenvolvimento de projetos de circuitos eletrônicos. 18.ed. São Paulo: Érica, 2001.

HALLIDAY, D.; RESNICK, R.; WALKER, J. Fundamentos de Física. V. 2. 6.ed. Rio de Janeiro: LTC, 2003.

HALlIDAY, D.; RESNICK, R.; WALKER, J. Fundamentos de Física. v. 2. 9.ed. Rio de Janeiro: LTC, 2012.

HAYNIE, D. T. Biological Thermodynamics. v.1. Cambridge University Press, 2001.

Informações complementares dos autores:

Fabrício Pimenta Neto: licenciado em Física e Matemática e mestre em Ensino de Ciências e Matemática pela Universidade Cruzeiro do Sul. Atualmente é professor da FESP/UEMG, $e$ mail: fabricio.neto@fespmg.edu.br .

Mauro Sérgio Teixeira de Araújo: bacharel em Física pela Universidade de São Paulo e Licenciado em Física pela Universidade de São Paulo, Mestre em Física pela Universidade de São Paulo e Doutor em Física pela Universidade de São Paulo. Atualmente é professor Titular da Universidade Cruzeiro do Sul, lecionando na Graduação e em cursos de Especialização. Leciona, orienta e realiza pesquisas em cursos de Mestrado e Doutorado, e-mail: mstaraujo@uol.com.br.

Artigo recebido para avaliação em 01/04/2014 e aceito para publicação em 12/11/2014. 